\title{
Purified IgG from aquaporin-4 neuromyelitis optica spectrum disorder patients alters blood-brain barrier permeability
}

\section{Alvaro Cobo Calvo}

Hopital Neurologique et Neurochirurgical Pierre Wertheimer https://orcid.org/0000-0002-2574-0721

\section{Alvaro Cobo-Calvo}

Hopital Neurologique et Neurochirurgical Pierre Wertheimer

Anne Ruiz

Centre de Recherche en Neurosciences de Lyon

\section{Chloé Richard}

Centre de Recherche en Neurosciences de Lyon

\section{Sandrine Blondel}

Centre de Recherche en Neurosciences de Lyon

\section{Sylvie Cavagna}

Centre de Recherche en Neurosciences de Lyon

\section{Nathalie Strazielle}

Centre de Recherche en Neurosciences de Lyon

\section{Jean-François Ghersi-Egea}

Centre de Recherche en Neurosciences de Lyon

\section{Pascal Giraudon}

Centre de Recherche en Neurosciences de Lyon

Romain Marignier ( $\nabla$ romain.marignier@chu-lyon.fr )

\section{Research}

Keywords: aquaporin-4 antibodies, blood-brain barrier, neuromyelitis optica

Posted Date: December 19th, 2019

DOI: https://doi.org/10.21203/rs.2.19310/v1

License: (c) (i) This work is licensed under a Creative Commons Attribution 4.0 International License.

Read Full License 


\section{Abstract}

Background: Blood-brain barrier (BBB) breakdown is considered one of the key steps for the development and lesion formation of neuromyelitis optica spectrum disorder (NMOSD). However, little is known about the molecular mechanisms involved. The aim of the study was to evaluate the effect of human immunoglobulins from NMOSD patients (NMO-IgG) on BBB properties.

Methods: Freshly isolated brain microvessels (IBM) from rat brains that mirror the blood-CNS barrier were used as a study model. At first, analysis of the secretome profile (cytokine protein array) from IBM exposed to purified NMO-IgG, to healthy donor IgG (Control-lgG), or non-treated, was performed. Second, structural BBB properties were analysed by Western blotting $(\mathrm{Wb})$ to measure the expression of tight junction (TJ) proteins (Claudin-5, occludin, and ZO-1) in fresh IBM and primary cultures of brain microvascular endothelial cells (BMEC) after exposition to NMO-IgG and Control-lgG. Finally, functional BBB properties were investigated using two approaches; 1 ) in the NMO-rat model, the presence of rat-IgG in tissue lysate from brain periventricular regions was analysed using $\mathrm{Wb}$, and 2) in a bicameral model simulating the blood and brain compartments of the BBB, the passage of NMO-IgG and sucrose was assessed by using ELISA.

Results: We found that NMO-IgG induces several functional and morphological changes of the BBB in our different study models, including: 1) increase of pro-inflammatory cytokines production (CXCL-10 [IP-10], IL-6, IL1-ra, IL1- $\beta$ and CXCL-3) by at least three-fold in IBM when exposed to NMO-IgG in comparison to Control-IgG or non-treated IBM; 2 ) decrease of Claudin- 5 levels by $25.6 \%$ after treatment of fresh IBM by NMO-IgG compared to Control-IgG ( $p=0.002)$, and similarly, decrease of Claudin- 5 by at least $20 \%$ when BMEC were cultured with NMO-IgG from five different patients; 3 ) a higher level of rat-IgG accumulated in periventricular regions of NMO-rats compared to Control-rats and an increase the permeability of BBB after NMO-IgG treatment in the bicameral model.

Conclusion: Human NMO-IgG induces both structural and functional alterations of BBB properties, suggesting a direct role of NMO-IgG on modulation of BBB permeability in NMOSD.

\section{Background}

Neuromyelitis optica spectrum disorders (NMOSD) is a severe autoimmune disease of the central nervous system (CNS) that mainly affects the optic nerve and the spinal cord. The discovery of a serum antibody, termed NMO-IgG, directed against the aquaporin4 channel expressed at the CNS interfaces, has enhanced the understanding of NMOSD which is now considered an autonomous entity with distinctive pathophysiology, different from multiple sclerosis [1].

Contrary to the extended evidence concerning NMO-IgG involvement in NMOSD tissue lesion formation, the mechanisms for antibody penetration into the CNS, still awaits elucidation. Over the last few years, four main hypotheses have been proposed to explain the passage of NMO-IgG, from either serum or cerebrospinal fluid (CSF) into CNS. First, NMO-IgG has been observed to access the brain from the blood 
(blood-CNS barrier) through fenestrated endothelial cells in circumventricular organs such as the area postrema where AQP4 protein is highly expressed [2,3]. Although, NMO-IgG deposition was initially thought to be restricted to the area postrema [2], a recent study, using a monoclonal murine AQP4antibody with high antigen affinity, found a wide diffusion in the CNS [4]. Second, in vitro models have shown that either the NMO-IgG itself [5,6], or other components from the serum of NMOSD patients (such as matrix-metalloproteinase $2 / 9$ protein, antibodies against brain endothelial cells, or glucose-regulated protein-78) may alter the blood brain barrier (BBB) at the glio-vascular unit (blood-CNS barrier) [7-9]. Third, NMO-IgG might gain access to the CNS via the CSF using the paravascular pathway (CSF-CNS barrier) [10] by which the end-feet of astrocytes would be directly exposed to CSF circulating NMO-IgG, allowing their entrance into the CNS [11]. In fact, higher levels of NMO-IgG have been found in the CSF of NMOSD patients at relapse compared to remission phases [12]. Finally, extravasation of the antibody through meningeal and small parenchymal vessels has been recently proposed as new route for antibody entry into the CNS [4].

In the field of NMOSD, the impact of NMO-IgG on BBB has mainly been evaluated using in vitro assays [5-8]. However, the use of ex vivo models could provide a more precise representation of the antibody behaviour when reaching the BBB, and a better understanding of the underlying pathophysiology.

Herein, an ex vivo approach consisting of fresh brain microvessels isolated from rat brain to model the blood-CNS barrier was used for the first time to assess the impact of NMO-IgG on the BBB. A first analysis was performed to determine whether IgG from AQP4-positive NMOSD patients (NMO-IgG) may orchestrate BBB alteration by inducing a specific cytokine secretome profile in isolated brain microvessels (IBM). Then, using both IBM and an in vivo animal model simulating CSF-CNS barrier, the structural modifications of the BBB induced by NMO-IgG were investigated. Finally, to evaluate whether such structural modifications are sufficient to induce a breakdown of the barrier an in vitro bicameral model was set-up to simulate the blood-CNS barrier.

\section{Methodology}

\section{Patients, IgG-patient purification, and NMO-IgG selection}

De-identified serum specimens were obtained from six different relapsing NMOSD patients that were included in the French cohort of NMOSD (NOMADMUS), and stored at NeuroBioTec (Biological Resource Centre of the Hospices Civils de Lyon, France). All patients were tested positive for AQP4-antibodies detected by cell-based assay [13], and all patients fulfilled the 2015 criteria for NMOSD [14]. Serum provided by the French blood service (Etablissement Français du Sang) from healthy blood donors were used as controls. Both NMO-IgG (called NMO-IgG ${ }_{1-6}$ ) and healthy donors-lgG (called Control-lgG) were purified from the NMOSD and healthy donor serums, respectively, using chromatography over Protein-A Sepharose as previously described [15]. IgGs were then used at a final concentration of $2 \mu \mathrm{g} / \mu \mathrm{l}$. 
NMO-IgG from the different patients were first tested on cultured astrocytes in order to select NMO-IgG for the experiments on fresh IBM and for the animal model (NMO-rat). After 24 hours of treatment with NMO$\lg _{1-6}$ (number of experiments=39), there was a significant decrease in cultured astrocyte AQP4 expression (mean 114.9 \pm 24.3$)$ when compared to treatment with Control-lgG $(n=12$, mean 70.3 \pm 17.9 , $p<0.001)$, as measured by western blot $(\mathrm{Wb})$. The effects of $\mathrm{NMO}^{-\operatorname{lgG}_{1-6}}$ and Control-lgG were evaluated in duplicate or triplicate. $\mathrm{NMO}^{-\lg \mathrm{G}_{1,2,6}}$ were the most pathogenic, inducing a higher decrease in AQP4

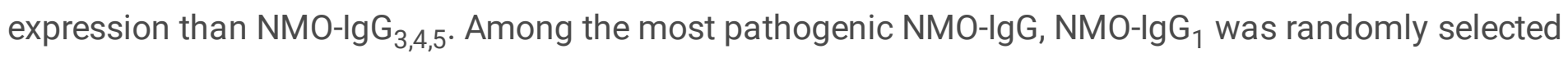
to carry out experiments.

\section{Intraventricular brain NMO-IgG infusion, and ethical procedures}

Animal surgery was performed as previously described [15]. Briefly, rats were anesthetised and mounted in a stereotaxic frame. Two hundred $\mu \mathrm{l}$ of purified $\mathrm{IgG}\left(\mathrm{NMO}^{-\mathrm{IgG}_{1}}\right)$ or $\mathrm{NaCL}$ (Control-IgG) were infused into the CSF during seven days at a $1 \mu \mathrm{L} /$ hour using a sterile subcutaneous osmotic pump. After seven days, rats were anesthetised and received an intracardiac perfusion of $100 \mathrm{ml}$ phosphate-buffered saline (PBS; $0.1 \mathrm{M}, \mathrm{pH}$ 7.4). Then, rats were sacrificed using pentobarbital, brain was then removed and frozen in isopentane at $-30^{\circ} \mathrm{C}$, and further stored at $-80^{\circ} \mathrm{C}$.

Brain tissue was embedded in Tissue-Tek OCT, and subsequently cut on a cryomicrotome [15]. Brain slices were used for immunohistochemical (IHC) study of tight junction proteins.

A total of 7 rats received $\mathrm{NMO}^{-\mathrm{IgG}_{1}}$ (NMO-rats) and 9 rats received $\mathrm{NaCl}$ (Control-rats) for different experiments. Animal care and procedures were carried out in accordance with the European Directive 2010/63/UE and followed the Animal Research Reporting of In Vivo Experiments (ARRIVE) guidelines. The study was approved by the local Lyon 1 University Animal Care Committee (B2012-80 project).

\section{Isolation of brain microvessels}

Isolation of brain microvessels was performed from two adult rat brains per experiment according to the procedure described [16]. Adult rats were anesthetised with isoflurane and decapitated. Brains were excised and placed at $4^{\circ} \mathrm{C}$ in Krebs- Ringer buffer (in $\mathrm{Mm}: 135 \mathrm{NaCl}, 4 \mathrm{KCl}, 2.2 \mathrm{CaCl}_{2}, 1.2 \mathrm{MgCl}_{2}, 6 \mathrm{NaHCO}_{3}$, 10 Hepes, 5 Glucose monohydrate, $\mathrm{pH}$ 7.4). Meninges and choroid plexuses were dissected. Using oxygen-saturated buffers, cortices were homogenised in a Dounce-type glass-glass homogeniser. The preparation was further homogenised in $5 \mathrm{vol} / \mathrm{g}$ tissue of $1 \%$ BSA-supplemented Kreps Ringer buffer (AKRB), and then filtered through a $500 \mu \mathrm{m}$ mesh sieve (Netwell, Corning, Corning, NY). The filtrate was diluted with 1\% AKRB (1:1) and homogenised again. Then, the homogenate was centrifuged for 10 minutes, the pellet was suspended in Krebs-Ringer buffer containing 17.5\% 70 kD-dextran, and further centrifuged for 27 minutes. Myelin was retained at the surface of the gradient and the resulting pellet was suspended in 1\% AKRB. Pellet was consecutively filtered through 200 and 74- $\mu \mathrm{m}$ mesh sieves to eliminate larger vessels. The preparation was centrifuged for 15 minutes. Finally, the pellet was 
suspended and filtered on a 40 $\mu \mathrm{m}$ mesh sieve (BD Bioscience, Erembodegem, Belgium). All procedures were performed at $4^{\circ} \mathrm{C}$.

The fresh IBM retained were recovered in AKRB $0.1 \%$ and $2 \mu$ were used to observe their morphology under the microscope. Immunofluorescence analysis showed the presence of Claudin-5, Occludin, and ZO-1 in fresh IBM preparation (Supplementary Figure 1). Fresh IBM suspended in $0.1 \%$ AKRB were centrifuged for $15 \mathrm{~min}$ at $800 \mathrm{rpm}$, and immediately suspended in $2 \mathrm{ml}$ of Dulbecco's Modified Eagle's $(D M E M)+B S A 0.1 \%$. To determine the ex vivo effect of NMO-IgG on tight junction proteins (Claudin-5, Occludin, and ZO-1), fresh IBM together with DMEM/ BSA 0.1\% were distributed in 6 different 24-well plates $(330 \mu \mathrm{l}$ in each well) and further treated by adding either $330 \mu \mathrm{l}$ of DMEM/BSA $0.1 \%$ (called nontreated IBM), Control-IgG, or NMO-Ig $G_{1}$. Fresh IBM were treated in duplicate and incubated for 20 hours at $37^{\circ} \mathrm{C}$. Finally, fresh IBM from each well were suspended in DMEM up to $1 \mathrm{ml}$.

Fresh IBM were prepared for Wb or cytokine/chemokine analysis after 20 hours of incubation.

\section{Brain microvascular endothelial cells}

Brain microvascular endothelial cells (BMEC) were isolated as previously described [17]. Briefly, microvessels were isolated from 3 rat brains following mechanical and enzymatic digestion of brain tissue, and plated in a T75 flask coated with collagen and fibronectin (microvessels from 1.5 cortex per T75 flask). BMEC were grown and purified with decreasing concentrations of puromycin for 5 days, followed by 3 days of culture in an insulin, transferrin, and sodium selenite-containing medium. The cells were cultured on plastic for BMEC experiments or harvested for seeding on filters to generate in vitro BBB models, as follows. After dissociation during 30 seconds with trypsin $0.05 \%$-EDTA $0.02 \%$ solution at $37^{\circ} \mathrm{C}$ per T75 flask, the detached BMEC (approximately $3^{*} 10^{6} \mathrm{cells} / \mathrm{T} 75$ ) were dissociated by pipetting up and down. Re-suspended BMEC were then added to the upper compartment of a pre-coated well (filters filled with endothelial cell media (ECM) at high density $\left(160 * 10^{3}\right.$ cells/filter of $\left.1.1 \mathrm{~cm}^{2}\right)$. Endothelial cells expressed tight junction proteins within 3 days [17]. BMEC were then exposed to DMEM/BSA0.1\% (termed non-treated BMEC), Control-lgG or $\mathrm{NMO}^{-\mathrm{IgG}_{1-6}}$ for 20 hours at the concentration of $350 \mu \mathrm{g} / \mathrm{ml}$.

\section{Permeability analyses: Bicameral model}

For antibody permeability analyses, BMEC were exposed to three different NMO-IgG in the upper compartment of the culture system. The acceptor compartment was then collected, and frozen for later NMO-IgG analysis by Enzyme-linked immunosorbent assays (ELISA). The apical part of the model simulates blood and the basolateral side simulates the CSF.

To test the integrity of the barrier, [14C] sucrose was added in the upper compartment at the end of the incubation with IgG, and sucrose transfer was measured over a 1-hour period. The radioactivity was measured over time in the lower compartment using a Perkin Elmer TRICARB, 4910TR liquid scintillation analyser, Singapore. Clearance curves were generated and permeability coefficients were calculated as described [18]. 


\section{Enzyme-linked immunosorbent assays}

The level of human IgG were quantified in the compartment acceptor by ELISA set according to the manufacturer's protocols (Human IgG ELISA Quantitation Set, Bethyl Laboratories, Inc, Montgomery, Alabama, USA). Samples were diluted at 1:15. The optical density was measured at $450 \mathrm{~nm}$ with $\lambda$ correction of $570 \mathrm{~nm}$ using a Spark spectrophotometric microplate reader (Tecan Trading AG, Switzerland). Based on the optical density value of each sample, the sample concentration was calculated in $\mathrm{ng} / \mathrm{mL}$ and used to calculate clearance.

\section{Cytokine Array analysis}

Evaluation of cytokines and chemokines from fresh IBM supernatants were assayed by using Proteome Profiler Array kit (rat cytokine array panel A, R\&D Systems, Minneapolis, MN, USA) according to the manufacturer's instruction. Fluorescence signal intensity was measured by ImageJ software.

\section{Astrocyte cultures}

As previously described, [15] primary glial cultures were isolated from 1-day-old rat pups ( $n=24)$ and dissociated cells were further diluted in DMEM to a density of $2.10^{5}$ cells. Cells were seeded in 6 -well plates and incubated at $37^{\circ} \mathrm{C}$ in a moist $5 \% \mathrm{CO}_{2}, 95 \%$ air atmosphere. In order to obtain pure astrocyte cultures and eliminate microglia and oligodendrocytes, cells were treated with cytosine arabinoside (AraC, 25nM, Sigma-Aldrich).

\section{Immunohistochemistry procedures}

Both cytospined IBM and brain slides were fixed in 4\% paraformaldehyde for 10 minutes, then washed in PBS, and blocked for 1 hour in blocking solution (10\% Normal Goat serum, PBS 1X, BSA 1\%, Triton 0.3\%). Then, the material was incubated overnight at $4^{\circ} \mathrm{C}$ with primary antibodies in blocking solution and further washed in PBS. Slides were labelled at room temperature with specific fluorescent secondary antibodies in blocking solution for 30 minutes, washed in PBS and incubated with 4',6-diamidino-2phenylindole (DAPI). Slides were mounted in tamponed glycerol and stored at $4^{\circ} \mathrm{C}$. Axio Imager Z1 Apotome technology (Zeiss, Oberkochen, Germany) was used to image the slides.

\section{Immunoblotting}

Fresh IBM, BMEC, or brain tissues were dissociated by ultrasound fragmentation in homogenisation buffer and phosphatases inhibitors, and then protein content was counted [15]. Protein samples were separated by sodium dodecyl sulphate-polyacrylamide gel electrophoresis (SDS-PAGE gels) and transferred to nitrocellulose membranes. Beta-actin was used as an internal standard. The membranes were treated with blocking buffer for 1 hour and incubated with the specified primary antibodies at $4^{\circ} \mathrm{C}$ overnight. Membranes were exposed to an anti-lgG antibody for 1-h at room temperature and revealed using chemiluminescence, as described [15]. Quantification of the band intensity was obtained using ImageJ software. 


\section{Immunohistochemical reagents}

The following lgGs were used for tissue sample immunodetection and lysates. The primary antibodies were; Claudin-5 (mouse monoclonal, 35-2500; Invitrogen), Occludin (rabbit polyclonal, 71-1500; Invitrogen), ZO-1 (rabbit polyclonal, 61-7300; Invitrogen), PDGF Receptor- $\beta$ (rabbit monoclonal, 28E1; Cell Signalling Technology), AQP4 (rabbit polyclonal AB2218; Merck@ KGaA); beta-Actin (mouse monoclonal, A1978; Sigma-Aldrich $\left.{ }^{\circledR}\right)$. The secondary antibodies were: Peroxidase-AffiniPure $F(a b) 2$ fragment donkey anti-mouse IgG (Heavy,[H]+light [L] chains) (715-036-151, Jackson Immunoresearch), Alexa Fluor®488 goat anti-mouse IgG, A11029; Alexa Fluor ${ }^{\circledR} 555$ goat anti-mouse IgG, A21424; Alexa Fluor ${ }^{\circledR} 488$ goat antirabbit lgG, A11034; and Alexa Fluor®455 goat anti-rabbit IgG, A21429 from Molecular Probes Inc.

\section{Statistical analysis}

All $\mathrm{Wb}$ analyses were performed by investigators blinded for treatment conditions. Data are presented as median (interquartile range [IQR]) and percentages. The non-parametric Kruskal-Wallis test was used to evaluate differences in more than two groups and U-Mann-Whitney test was used to compare two groups. All statistical analyses were performed using Prism 5.0 GraphPad Inc software.

\section{Results}

\section{Purified IgG from NMOSD patients induces an inflammatory secretome profile in brain microvessels}

Because soluble factors secreted by the BBB cells may auto-modulate BBB function in the presence of

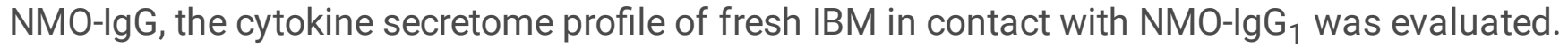

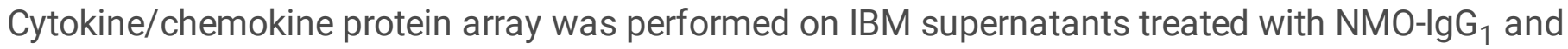
Control-IgG. Expression levels (mean fluorescence signal intensity) of IL-6, IL1-ra, IL1- $\beta$, CXCL10 (IP-10) and CXCL3 showed at least a three-fold increase in IBM exposed to $\mathrm{NMO}^{-I g G_{1}}$ compared to control conditions (non-treated fresh IBM or Control-IgG). For the ten following cytokines/chemokines, TNFa, RANTES, IL17, IL4, GMCSF, CNTF, MIP1 a, CX3CL1, CXCL1/2 and TIMP1, expression levels were at least two fold (Figure 1).

Globally, exposition to purified IgG from AQP4-antibody positive NMOSD patients resulted in the production of an inflammatory secretome profile from brain microvessels.

\section{Purified-IgG induce Claudin-5 loss in brain microvessels}

Assuming that the inflammatory environment induced by NMO-IgG has the capacity to alter the BBB, Claudin-5, Occludin, and ZO-1 protein expression analysis was performed in fresh IBM treated with NMO$\lg _{1}(n=6)$ and Control-IgG $(n=4)$ for 20 hours (Figure 2.a Table 1.a). Immunoblotting showed a

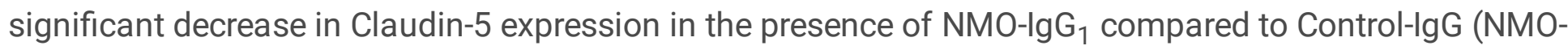
$\operatorname{lgG}_{1}, 25.61 \%$ vs. Control-IgG, 3.91\%; $\left.\mathrm{p}=0.002\right)$. There were no significant modifications regarding Occludin and ZO-1 expression. 
Using the NMO rat model [15] an in vivo expression analysis of the same tight junction proteins was performed using $\mathrm{Wb}$ on fresh IBM obtained from these animals. Claudin- 5 expression decreased by $16.2 \%$ in NMO-rat $(n=1)$, compared to Control-rat $(n=1)$ (Supplementary Table 1 ).

These findings were confirmed in primary BMEC cultures following 20-hour treatments with the six NMO$\operatorname{lgG}_{1-6}$ (Figure 2.b Table 1.b). Immunoblotting showed that Claudin-5 expression decreased by at least

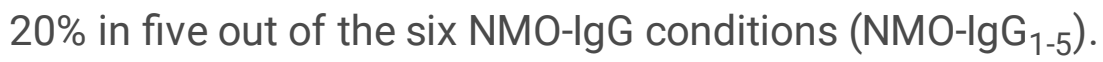

Tight junction proteins in situ, in the brain of NMO-rat were also studied. A decrease pattern of immunofluorescent staining intensity of periventricular Claudin-5 expression in NMO-rats $(n=2)$ compared to Control-rats ( $n=2)$ was found (Figure 3 ) but not for Occludin and ZO-1.Overall, these results indicate that purified IgG from AQP4-antibody positive NMOSD patients modify the expression of the tight junction protein Claudin-5.

When comparing the effects of each $\mathrm{NMO}^{-\operatorname{lgG}_{1-6}}$ on either AQP4 expression in astrocytes or on Claudin-5 on BMEC, expression changes in each cell type was not correlated (rho=-0.486, $\mathrm{p}=0.329$ ) (Figure 4).

On the bases of this observation, we evaluated whether IgG from the six tested NMOSD patients were directed to BMEC. Using Wb on BMEC lysate, we did not find any binding pattern neither with patients' IgG $\left(\mathrm{NMO}_{1-6}\right)$ nor Control-IgG (Supplementary Figure 2).

\section{NMO-IgG induce alteration of BBB functional properties in vivo and in vitro}

The observation, in ex vivo and in vivo experiments, that NMO-IgG induce structural modification on tight junction proteins, suggests that NMO-IgG may lead to an alteration of BBB functional properties. We evaluate the diffusion of rat-lgG in periventricular brain areas using immunoblotting on the brains of NMO-rats $(n=4)$ and Control-rats $(n=6)$. One extra rat (Control-animal) did not receive saline intracardiac perfusion which allows blood elimination from brain vasculature and was thus used to identify rat IgG using Wb. Heavy $[\mathrm{H}]$ and light $[\mathrm{L}]$ rat-lgG chains were well detected in the brain of the Control-animal. A higher proportion of NMO-rats (3 out of 4 rats) accumulated rat-IgG (mainly L chain) in the periventricular regions compared to Control-rat (only traces of $\mathrm{H}$ and $\mathrm{L}$ chains in 6 rats) (Figure 5).

These observations show that rat-IgG can cross the BBB, from the periphery to the CNS parenchyma, when the rat is chronically infused with NMO-IgG. This suggests that NMO-IgG present in the CSF compartment can modify BBB functional properties in the periventricular regions.

To further assess the effect of NMO-IgG on the BBB, we evaluated the functional properties of the BBB using a bicameral model simulating the blood-CNS. First, we measured the clearance of three NMO-IgG over 24 hours. NMO-IgG 2 clearance (median 1.22 [1.12-1.75]) was twice that of the Control-lgG (median $0.54 ;[0.35-0.73]) ; p=0.050)$. No other differences were found between the other NMO-IgG tested and the IgG-Control (Table 2). After exposing cells to the NMO-IgG or control-IgG, the permeability to sucrose was then measured over 3 hours. There was a significantly higher sucrose clearance rate in cells treated with 
NMO-IgG 1 (median 0.42; [0.36-0.51]) and NMO-IgG 2 (median 0.44; [0.41-0.48] than in those treated with Control-IgG (median 0.31; [0.29-0.34]; $p=0.050$ ) (Table 2). These results indicate that NMO-IgG added in the blood compartment weaken the BBB.

\section{Discussion}

The present study evaluating the effect of purified IgG from AQP4-antibody positive NMOSD patients on BBB properties showed that: 1) NMO-IgG induce an inflammatory secretome profile in brain microvessels, 2) have a direct impact on the molecular structure of the BBB, and 3) induce a functional alteration of the BBB.

In line with previous studies, we found an upregulation of several cytokines and chimokines, notably IL1ra, IL1- $\beta$, TNFa, CXCL10 and CXCL3. This inflammatory secretome has been found increased in the CSF or serum of NMOSD patients reflecting a cell recruitment to sites of inflammation or even BBB weakening [19-23]. Interestingly, we found an up-regulation of IL-6, proposed to be a surrogate diagnostic and prognostic marker in NMOSD [19,24-26], enhancing plasmablast survival and AQP4-antibody secretion [27]. More recently, IL-6 receptor blockage treatments have been proposed as a promising therapeutic option for NMOSD $[28,29]$. Apart from its effect on plasmablasts, IL-6 could be involved in BBB alteration. Indeed, a recent study using astrocyte and endothelial cell co-cultures showed IL-6 production in astrocytes and AQP4 internalisation after exposure to NMO-IgG in the abluminal side of the culture (brain compartment) [5]. Authors also found a structural BBB impairment characterised by a decreased and discontinuous Claudin- 5 immunoreactivity at tight junctions, and a molecular leakage through the BBB that was reverted after IL-6 soluble receptor blocking [5]. Our data suggest that isolated capillaries may be a source of IL-6 production other than astrocytes, as previously described [20,30]. In summary, the specific inflammatory secretome induced by NMO-IgG in brain microvessels likely modulates a subsequent pathophysiological process characterised by the structural weakening and functional impairment of the BBB. Whether endothelial cells or pericytes remaining embedded in the basal membrane that delimit the isolated microvessels are the main source of these cytokines remains to be established.

In order to study the impact of NMO-IgG on BBB structure itself, we use an original ex vivo BBB mimicking the close relationship between NMO-IgG and brain microvessels. Fresh IBM are known to maintain in vivo BBB properties, and have been previously used for the study of endothelial molecular transporters [31,32], drug pharmacokinetic or pharmacodynamics [33], and disease pathophysiology in animal models [34,35]. However, the application of this technique on NMOSD-related models has not been previously performed. The decrease in expression of Claudin-5 in IBM when exposed to NMO-IgG in the absence of any previous contact with breaching substances, suggests that NMO-IgG directly contribute to the structural destabilization of the BBB from the brain compartment. Moreover, we further reproduced same findings with the use of BMEC cultured with NMO-IgG observing such decrease of Claudin-5 expression. Two previous studies found similar results with a down-regulation of Claudin-5 in immortalised human brain microvascular endothelial cells treated with the serum of NMO patients but not in controls $[7,8]$. 
Accordingly, a decrease in the transendothelial electrical resistance (TEER) was observed which suggested a functional disruption of the BBB. $[7,8]$

Whether the NMO-IgG "by itself" or other unknown auto-antibodies present in NMOSD patients are able to directly open the BBB is not completely resolved. Interestingly, we found that the decreased expression of Claudin- 5 did not correlate with the loss of AQP4 in individual patients. This finding may suggest that other IgG, different from the specific AQP4-antibody, could be involved in BBB disruption as recently proposed [7-9]. In fact, GRP78 an antibody specific to the surface of endothelial proteins has been recently identified in the serum of patients with AQP4-antibodies and lupus erythematous systemic [9]. Other study described antibodies against BMEC in the serum of NMOSD patients that could alter BBB properties through an autocrine secretion of antivascular endothelial growth factor (VEGF) by the BMEC. [7] However, when performing the Wb we did not observe any specific pattern of IgG binding to endothelial cells in the present study.

NMO-IgG may exert its pathological effect from the CSF compartment after passing through subpial spaces where vessels penetrate the brain parenchyma $[4,11]$. To validate this hypothesis in vivo, an intraventricular chronic infusion of NMO-IgG in rat brain was performed. This leads to a wide NMO-IgG distribution through CSF in the neural tissues [15], reaching the subarachnoid and subpial spaces [11]. By analysing brain microvessels from NMO-rats it was possible to detect not only the deleterious effect of NMO-IgG, concerning mainly the loss of Claudin- 5 but also a functional alteration represented by the passage of rat-IgG from the blood to the CNS. AQP4-expressing astrocytic end-feet would be directly exposed to CSF NMO-IgG, thus triggering astrocyte damage and further BBB disruption [6].

\section{Conclusion}

Overall, the present study provides a global perspective on the pathophysiological role of NMO-IgG at the BBB level. NMO-IgG induce a distinctive inflammatory secretome profile that may modulate the subsequent alterations of the BBB properties. The major finding involved in structural weakening is driven by the decrease of Claudin-5 expression both from the blood and the CSF compartments. The functional alteration is reflected by a higher permeability through brain microvessels allowing the passage of circulating molecules to the CNS when the antibody is present in the CSF compartment. To this regard, the NMO-IgG "by itself" may penetrate through the breaching barrier, distribute through the extracellular fluid and, finally, trigger the disease after binding to the AQP4 channel.

\section{Declarations}

\section{Author's contributions}

AC-C, AR, NS, JF G-E, PG and RM contributed to model design, implemented the model, performed simulations, and were major contributors to writing the manuscript. CR, SR and SC contributed to perform simulations and the critical review of the manuscript. 
Acknowledgements

A. Cobo-Calvo was supported by a grant from the Fundación Alfonso Martín Escudero for this study. The authors thank the group of NeuroBioTec from Hospices Civils de Lyon for supporting this study.

\section{Competing interests}

The authors declare that they have no competing interests

\section{Availability of data and materials}

The datasets used and/or analysed during the current study are available from the corresponding author on reasonable request.

\section{Consent for publication}

N/A.

\section{Ethics approval and consent to participate}

N/A.

\section{Funding}

The present study is supported by a grant from ARSEP foundation and a grant provided by the French State and handled by the "Agence Nationale de la Recherche", within the framework of the "Investments for the Future" programme, under the reference ANR-10-COHO-002 Observatoire Français de la Sclérose en Plaques (OFSEP), and the Eugene Devic Foundation against Multiple Sclerosis (EDMUS Foundation.

\section{Abbreviations}

Neuromyelitis optica spectrum disorders: NMOSD; central nervous system: CNS; cerebrospinal fluid: CSF; blood brain barrier: BBB; isolated brain microvessels: IBM; western blot: Wb; phosphate-buffered saline: PBS; immunohistochemical: IHC; BSA-supplemented Kreps Ringer buffer: AKRB; Dulbecco's Modified Eagle's: DMEM; Enzyme-linked immunosorbent assays: ELISA; 4',6-diamidino-2-phenylindole: DAPI; interquartile range: IQR; antivascular endothelial growth factor: VEGF.

\section{References}

1. Misu T, Höftberger R, Fujihara K, Wimmer I, Takai Y, Nishiyama S, et al. Presence of six different lesion types suggests diverse mechanisms of tissue injury in neuromyelitis optica. Acta Neuropathol 2013;125:815-27.

2. Ratelade J, Bennett JL, Verkman AS. Intravenous neuromyelitis optica autoantibody in mice targets aquaporin-4 in peripheral organs and area postrema. PLoS One. 2011;6. 
3. Miyata S. New aspects in fenestrated capillary and tissue dynamics in the sensory circumventricular organs of adult brains. Front. Neurosci. 2015;9:390.

4. Hillebrand S, Schanda K, Nigritinou M, Tsymala I, Böhm D, Peschl P, et al. Circulating AQP4-specific auto-antibodies alone can induce neuromyelitis optica spectrum disorder in the rat. Acta Neuropathol. 2019;137:467-485.

5. Takeshita Y, Obermeier B, Cotleur AC, Spampinato SF, Shimizu F, Yamamoto E, et al. Effects of neuromyelitis optica-IgG at the blood-brain barrier in vitro. Neurol Neuroimmunol neuroinflammation 2017;4:e311.

6. Vincent T, Saikali P, Cayrol R, Roth AD, Bar-Or A, Prat A, et al. Functional consequences of neuromyelitis optica-IgG astrocyte interactions on blood-brain barrier permeability and granulocyte recruitment. J Immunol 2008;181:5730-5737.

7. Shimizu F, Sano Y, Takahashi T, Haruki H, Saito K, Koga M, et al. Sera from neuromyelitis optica patients disrupt the blood-brain barrier. J Neurol Neurosurg Psychiatry 2012;83:288-297.

8. Tasaki A, Shimizu F, Sano Y, Fujisawa M, Takahashi T, Haruki H, et al. Autocrine MMP-2/9 secretion increases the BBB permeability in neuromyelitis optica. J Neurol Neurosurg Psychiatry 2014;85:419430.

9. Shimizu F, Schaller KL, Owens GP, Cotleur AC, Kellner D, Takeshita Y, et al. Glucose-regulated protein 78 autoantibody associates with blood-brain barrier disruption in neuromyelitis optica. Sci Transl Med 2017;9:397.

10. Iliff JJ, Wang M, Liao Y, Plogg BA, Peng W, Gundersen GA, et al. A Paravascular Pathway Facilitates CSF Flow Through the Brain Parenchyma and the Clearance of Interstitial Solutes, Including Amyloid. Sci Transl Med 2012;4:147ra111.

11. Asgari N, Berg CT, Mørch MT, Khorooshi R, Owens T. Cerebrospinal fluid aquaporin-4-immunoglobulin G disrupts blood brain barrier. Ann Clin Transl Neurol 2015;2:857-863.

12. Sato DK, Callegaro D, De Haidar Jorge FM, Nakashima I, Nishiyama S, Takahashi T, et al. Cerebrospinal fluid aquaporin-4 antibody levels in neuromyelitis optica attacks. Ann Neurol. 2014;76:305-309.

13. Marignier R, Bernard-Valnet R, Giraudon P, Collongues N, Papeix C, Zéphir H, et al. Aquaporin-4 antibody-negative neuromyelitis optica: distinct assay sensitivity-dependent entity. Neurology 2013;80:2194-200.

14. Wingerchuk DM, Banwell B, Bennett JL, Cabre P, Carroll W, Chitnis T, et al. International consensus diagnostic criteria for neuromyelitis optica spectrum disorders. Neurology 2015;85:177-89.

15. Marignier R, Ruiz A, Cavagna S, Nicole A, Watrin C, Touret M, et al. Neuromyelitis optica study model based on chronic infusion of autoantibodies in rat cerebrospinal fluid. J Neuroinflammation 2016;13:111.

16. Gazzin S, Strazielle N, Schmitt C, Fevre-Montange M, Ostrow JD, Tiribelli C, et al. Differential expression of the multidrug resistance-related proteins $A B C b 1$ and $A B C c 1$ between blood-brain interfaces. J Comp Neurol 2008;510:497-507. 
17. Molino Y, Jabès F, Lacassagne E, Gaudin N, Khrestchatisky M. Setting-up an in vitro Model of Rat Blood-brain Barrier (BBB): A Focus on BBB Impermeability and Receptor-mediated Transport. J Vis Exp 2014;88:e51278.

18. Strazielle N, Preston JE. Transport across the choroid plexuses in vivo and in vitro. Methods Mol Med 2003;89:291-304.

19. Uzawa A, Mori M, Arai K, Sato Y, Hayakawa S, Masuda S, et al. Cytokine and chemokine profiles in neuromyelitis optica: Significance of interleukin-6. Mult Scler. 2010;16:1443-52.

20. Shimizu F, Nishihara H, Sano Y, Takeshita Y, Takahashi S, Maeda T, et al. Markedly increased IP-10 production by blood-brain barrier in neuromyelitis optica. PLoS One. 2015;10.

21. Barros PO, Linhares UC, Teixeira B, Kasahara TM, Ferreira TB, Alvarenga R, et al. High in vitro immune reactivity to Escherichia coli in neuromyelitis optica patients is correlated with both neurological disabilities and elevated plasma lipopolysaccharide levels. Hum Immunol. 2013;74:1080-7.

22. Sozen T, Tsuchiyama R, Hasegawa Y, Suzuki H, Jadhav V, Nishizawa S, et al. Role of interleukin1 beta in early brain injury after subarachnoid hemorrhage in mice. Stroke 2009;40:2519-25.

23. Fife BT, Kennedy KJ, Paniagua MC, Lukacs NW, Kunkel SL, Luster a D, et al. CXCL10 (IFN-gammainducible protein-10) control of encephalitogenic CD4+ T cell accumulation in the central nervous system during experimental autoimmune encephalomyelitis. J Immunol. 2001;166:7617-24.

24. Wang Y, Zhou Y, Sun X, Lu T, Wei L, Fang L, et al. Cytokine and Chemokine Profiles in Patients with Neuromyelitis Optica Spectrum Disorder. Neuroimmunomodulation 2016;23:352-8.

25. Uzawa A, Mori M, Sawai S, Masuda S, Muto M, Uchida T, et al. Cerebrospinal fluid interleukin-6 and glial fibrillary acidic protein levels are increased during initial neuromyelitis optica attacks. Clin Chim Acta 2013;421:181-3.

26. Barros PO, Cassano T, Hygino J, Ferreira TB, Centurião N, Kasahara TM, et al. Prediction of disease severity in neuromyelitis optica by the levels of interleukin (IL)-6 produced during remission phase. Clin Exp Immunol 2016;183:480-9.

27. Chihara N, Aranami T, Sato W, Miyazaki Y, Miyake S, Okamoto T, et al. Interleukin 6 signaling promotes anti-aquaporin 4 autoantibody production from plasmablasts in neuromyelitis optica. Proc Natl Acad Sci U S A. 2011;108:3701-6.

28. Araki M, Matsuoka T, Miyamoto K, Kusunoki S, Okamoto T, Murata M, et al. Efficacy of the anti-IL-6 receptor antibody tocilizumab in neuromyelitis optica: a pilot study. Neurology 2014;82:1302-6.

29. Yamamura T, Kleiter I, Fujihara K, Palace J, Greenberg B,Zakrzewska-Pniewska B, et al. Trial of Satralizumab in Neuromyelitis Optica Spectrum Disorder. N Engl J Med 2019;381:2114-2124.

30. Kaplin AI, Deshpande DM, Scott E, Krishnan C, Carmen JS, Shats I, et al. IL-6 induces regionally selective spinal cord injury in patients with the neuroinflammatory disorder transverse myelitis. $\mathrm{J}$ Clin Invest. 2005;115:2731-41.

31. Al Feteisi H, Al-Majdoub ZM, Achour B, Couto N, Rostami-Hodjegan A, Barber J. Identification and Quantification of Blood-Brain Barrier Transporters in Isolated Rat Brain Microvessels. J Neurochem 2018;146:670-685. 
32. Gazzin S, Strazielle N, Schmitt C, Fevre-Montange M, Ostrow JD, Tiribelli C, et al. Differential expression of the multidrug resistance-related proteins $A B C b 1$ and $A B C c 1$ between blood-brain interfaces. J Comp Neurol. 2008;510:497-507.

33. Schaefer CP, Arkwright NB, Jacobs LM, Jarvis CK, Hunn KC, Largent-Milnes TM, et al. Chronic morphine exposure potentiates p-glycoprotein trafficking from nuclear reservoirs in cortical rat brain microvessels. PLoS One. 2018;13.

34. Langdon KD, Cordova CA, Granter-Button S, Boyd JD, Peeling J, Murphy TH, et al. Executive dysfunction and blockage of brain microvessels in a rat model of vascular cognitive impairment. $J$ Cereb Blood Flow Metab 2018:38:1727-1740.

35. Ly H, Verma N, Wu F, Liu M, Saatman KE, Nelson PT, et al. Brain microvascular injury and white matter disease provoked by diabetes-associated hyperamylinemia. Ann Neurol. 2017;82:208-222.

\section{Table}

Table 1.a Tight junction protein expression in freshly isolated brain microvessels after 20 hours of NMOIgG treatment

\begin{tabular}{lccc}
\hline $\begin{array}{l}\text { Tight junction } \\
\text { proteins }\end{array}$ & \%expression/non- treated IBM & \%change/non- treated IBM & p-value \\
\hline Claudin-5 & & & \\
\hline Control-IgG & 96.09 & -3.91 & 1.0 \\
NMO-IgG $_{1}$ & 74.39 & -25.61 & 0.002 \\
\hline Occludin & & & \\
\hline Control-IgG & 85.09 & -14.91 & 0.150 \\
NMO-IgG $_{1}$ & 85.91 & -14.09 & 0.305 \\
\hline ZO-1 & & & \\
\hline Control-IgG & 96.33 & -3.7 & 1.0 \\
NMO-IgG $_{1}$ & 101.76 & +1.76 & 1.0 \\
\hline
\end{tabular}

Abbreviations; IBM, isolated brain microvessels; NMO, neuromyelitis optica

Table 1.b Tight junction protein expression changes in brain microvascular endothelial cells after 20 hours of NMO-IgG treatment 


\begin{tabular}{|c|c|c|c|c|c|c|}
\hline \multirow[b]{2}{*}{$\begin{array}{l}\text { IgG- } \\
\text { treatment }\end{array}$} & \multicolumn{2}{|c|}{ Claudin-5 } & \multicolumn{2}{|c|}{ Occludin } & \multicolumn{2}{|c|}{ ZO-1 } \\
\hline & $\begin{array}{c}\text { Ratio } \\
\text { Claudin5/actin }\end{array}$ & $\begin{array}{l}\% \text { change to } \\
\text { Control- IgG }\end{array}$ & $\begin{array}{c}\text { Ratio } \\
\text { Occludin/actin }\end{array}$ & $\begin{array}{l}\% \text { change to } \\
\text { Control-IgG }\end{array}$ & $\begin{array}{l}\text { Ratio } \\
\text { ZO- } \\
\text { 1/actin }\end{array}$ & $\begin{array}{c}\% \text { change } \\
\text { to Control- } \\
\text { IgG }\end{array}$ \\
\hline $\begin{array}{l}\text { Non- } \\
\text { treated }\end{array}$ & 1.33 & - & 1.95 & - & 1.05 & - \\
\hline $\begin{array}{l}\text { Control- } \\
\text { IgG }\end{array}$ & 1.23 & -7.97 & 2.63 & +34.87 & 1.17 & $+11,07$ \\
\hline $\begin{array}{l}\text { Pool } \\
\text { NMO-lgG } 1- \\
6\end{array}$ & 1.02 & -22.93 & 2.48 & +27.52 & 0.91 & -13.32 \\
\hline NMO-lgG 1 & 1.03 & -22.50 & 3.56 & +82.56 & 0.94 & -10.76 \\
\hline $\mathrm{NMO}^{-\mathrm{IgG}_{2}}$ & 1.02 & -23.70 & 2.30 & +17.75 & 0.96 & -8.47 \\
\hline $\mathrm{NMO}^{-l g G_{3}}$ & 1.01 & -24.28 & 2.19 & +12.22 & 0.98 & -6.84 \\
\hline $\mathrm{NMO}^{-\mathrm{IgG}_{4}}$ & 0.94 & -29.64 & 2.02 & +3.85 & 0.75 & -28.87 \\
\hline $\mathrm{NMO}^{-I g G_{5}}$ & 0.91 & -31.92 & 2.21 & +13.16 & 0.88 & -16.13 \\
\hline $\mathrm{NMO}^{-I g G_{6}}$ & 1.24 & -6.79 & 2.64 & +35.49 & 0.96 & -8.89 \\
\hline
\end{tabular}

Abbreviations; IBM, isolated brain microvessels; NMO, neuromyelitis optica

Table 2. Permeability of in vitro blood-brain barrier to immunoglobulins and sucrose

\begin{tabular}{l|ccccc}
\hline $\begin{array}{l}\text { Permeability, median } \\
\text { (IQR) }\end{array}$ & $\begin{array}{c}\text { Control- } \\
\text { IgG }\end{array}$ & NMO-IgG $_{1}$ & NMOIgG $_{2}$ & $\begin{array}{c}\text { NMO- } \\
\text { IgG }_{3}\end{array}$ & $\begin{array}{c}\text { p-value } \\
\text { (Kruskal } \\
\text { Wallis) }\end{array}$ \\
\hline IgG (ul.hr -1. cm-2) & 0.54 & 0.98 & 1.22 & 1.17 & 0.238 \\
& $(0.35-$ & $(0.54-$ & $(1.12-$ & $(0.30-$ & \\
Sucrose (ul.min -1. cm- & $0.73)$ & $1.45)$ & $1.75)^{*}$ & $1.18)$ & \\
$2)$ & 0.31 & 0.42 & 0.44 & 0.39 & 0.042 \\
& $(0.29-$ & $(0.36-$ & $(0.41-$ & $(0.33-$ & \\
& $0.34)$ & $0.51)^{*}$ & $0.48) *$ & $0.39)$ & \\
\hline
\end{tabular}

$* p=0.050$ vs. Control IgG

Abbreviation; IQR, interquartile range

\section{Figures}

Figure 1 
Cytokine/chemokine expression in isolated brain microvessels after 9 hours of treatment with NMOIgG1,Control-IgG and non-treated. *2 and **3-fold increase in fluorescence signal intensity in NMO-IgG1 vs. Control-IgG.or non- treated microvessels

\section{Figure 2}

Tight junction protein expression detected using Western blotting in ex vivo and in vivo models of NMOIgG treatment. 2.a Expression of tight junction proteins in freshly isolated brain microvessels treated for 20 hours with Control-IgG $(n=4)$ or NMO-IgG $(n=6)$ 2.b. Expression of tight junction proteins in brain microvascular endothelial cells after 20 hours of treatment with Control-lgG or NMO-IgG. *p-value $<0.05$ Non-treated freshly isolated brain microvessels (reference category) is depicted as red line

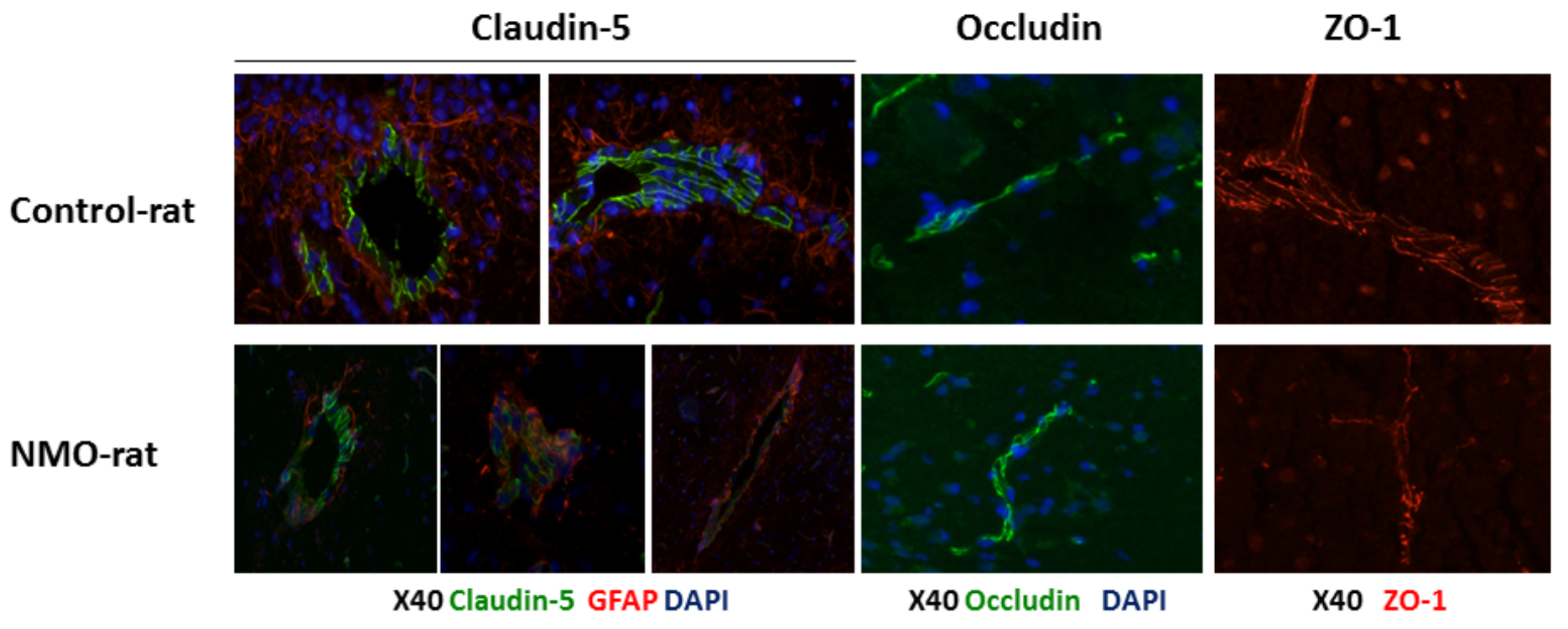

\section{Figure 3}

Tight junction protein expression in brain microvessels of periventricular areas after NMO-lgG intraventricular infusion 


\section{Figure 4}

Aquaporin-4 and Claudin-5 expression tested in astrocytes and brain microvascular endothelial cells, respectively.

\section{Figure 5}

Rat IgG in periventricular areas of CNS after seven days NMO-IgG and Control-IgG infusions. Immunodetection using Western Bloting of heavy $(\mathrm{H})$ and light $(\mathrm{L})$ immunoglobulin chains (black arrows) accumulated in neural tissues of chronically intraventricular infused NMO-rats (NMO-IgG) and Controlrats (Control-lgG). The Control sample did not receive the saline perfusion allowing blood elimination from brain vasculature, and as such was used to identify rat-IgG in nervous tissue. Actin was used as protein deposition control, same blot.

\section{Supplementary Files}

This is a list of supplementary files associated with this preprint. Click to download.

- SupplementaryTable1.docx

- SupplementaryFigure2....tif

- SupplementaryFigure1..tif

- LegendsFiguresSuppMaterial.docx 